\title{
Fecal Microbiota Transplantation: An Update on Clinical Practice
}

\author{
Kyeong $0 \mathrm{k} \mathrm{Kim}^{1,2}$ and Michael Gluck ${ }^{2}$ \\ ${ }^{1}$ Division of Gastroenterology and Hepatology, Department of Internal Medicine, Yeungnam University College of Medicine, Daegu, Korea, \\ ${ }^{2}$ Digestive Disease Institute, Virginia Mason Medical Center, Seattle, WA, USA
}

Fecal microbiota transplantation (FMT) is an infusion in the colon, or the delivery through the upper gastrointestinal tract, of stool from a healthy donor to a recipient with a disease believed to be related to an unhealthy gut microbiome. FMT has been successfully used to treat recurrent Clostridium difficile infection (rCDI). The short-term success of FMT in rCDI has led to investigations of its application to other gastrointestinal disorders and extra-intestinal diseases with presumed gut dysbiosis. Despite the promising results of FMT in these conditions, several barriers remain, including determining the characteristics of a healthy microbiome, ensuring the safety of the recipient with respect to long-term outcomes, adequate monitoring of the recipient of fecal material, achieving high-quality control, and maintaining reasonable costs. For these reasons, establishing uniform protocols for stool preparation, finding the best modes of FMT administration, maintaining large databases of donors and recipients, and assuring that oral ingestion is equivalent to the more widely accepted colonoscopic infusion are issues that need to be addressed. Clin Endosc 2019;52:137-143

Key Words: Fecal microbiota transplantation; Clostridium difficile infection; Colonoscopy

\section{INTRODUCTION}

Since the first modern descriptions of its use in 1958, ${ }^{1}$ fecal microbiota transplantation (FMT) has increasingly gained interest and rapid acceptance during the last 10 years. $^{2}$ FMT is defined as the infusion of stool from a healthy individual to a patient with presumed gut dysbiosis. ${ }^{3,4}$ FMT can also be delivered through an enteral route either via an endoscope, a nasoenteric tube, or capsules for ingestion. The presumed mechanism of action appears to be the establishment of a new gut microbiota community to restore the normal gut function..$^{5-7}$ On the basis of the concept of repopulating the gut with a healthy microbiome, FMT has been successfully used in the treatment of Clostridium difficile infection (CDI),

Received: December 8, 2018 Revised: January 10, 2019

Accepted: March 6, 2019

Correspondence: Michael Gluck

Digestive Disease Institute, Virginia Mason Medical Center, 1100 Ninth Ave,

C3-GAS, Seattle, WA 98101, USA

Tel: +1-206-223-2319, Fax: +1-206-341-1405,

E-mail: Michael.Gluck@virginiamason.org

ORCID: https://orcid.org/0000-0002-2048-3949

(c) This is an Open Access article distributed under the terms of the Creative Commons Attribution Non-Commercial License (http://creativecommons.org/ licenses/by-nc/3.0) which permits unrestricted non-commercial use, distribution, and reproduction in any medium, provided the original work is properly cited. and recommended for other conditions such as inflammatory bowel disease (IBD), autoimmune disorders, certain allergic diseases, and metabolic disorders such as obesity. ${ }^{8}$ In recurrent CDI (rCDI), the efficacy and safety of FMT has been proven by several randomized clinical trials (RCTs), and guidelines recommend the use of FMT as a second-line treatment. ${ }^{9-15}$ Success rates approaching $92 \%$ have been demonstrated in the treatment of rCDI. ${ }^{10,11}$

With the increasing use of FMT owing to its success in treating various diseases, there is growing demand for standardizing the preparation of fecal material, using accepted standards for the delivery, ensuring safety for the recipient, monitoring long-term outcomes, and continuously improving the procedural processes and safety.

\section{INDICATIONS AND CONSIDERATIONS FOR THE RECIPIENT}

The use of FMT became rapidly accepted mainly owing to its success in treating rCDI. Data from several RCTs and a large case series revealed the efficacy and safety of FMT. ${ }^{9-12}$ A recent systematic review and meta-analysis of 7 RCTs and 30 case series demonstrated the superiority of FMT over 
vancomycin therapy, with resolution rates as high as $92 \%{ }^{16}$ Moayyedi et al. showed that FMT was superior to placebo or vancomycin therapy. ${ }^{17}$ Fischer et al. reported that FMT clinically cured $91 \%$ of patients with severe rCDI that failed maximal medical therapy. ${ }^{18}$

The indications for FMT include mild to moderate rCDI after a second recurrence following treatment with a standard antibiotic, moderate rCDI that is not responding to standard therapy after 1 week, and severe CDI that is refractory to standard therapy after $48 \mathrm{~h} .{ }^{3,19}$ The recent U.S. guidelines recommend FMT in patients with 3 or more recurrences of CDI, although most clinicians prescribe FMT if CDI recurs after 2 courses of antibiotics. ${ }^{15}$ The European consensus guidelines suggest that FMT should be considered after the first episode if the disease is severe and refractory to the initial antibiotic therapy. ${ }^{20}$ Although there are no strict guideline recommendations stating that CDI should be initially treated with FMT instead of antibiotics, there may be special situations that would justify its application, such as inability to deliver antibiotics to a patient with severe disease, intolerance of the patient to antibiotics, or as a substitute for surgery in highly unstable patients. ${ }^{21}$

It has been suggested that gut dysbiosis underlies the pathogenesis of IBD. Zhang et $\mathrm{al}^{22}$ reported that in ulcerative colitis, there is decreased diversity in the patient's microbiota with fewer Firmicutes bacteria and more Proteobacteria. In a recently reported RCT on ulcerative colitis, the response rate to FMT was $55 \%$, with remission in $20 \%$ of the patients. ${ }^{23}$ Another RCT reported similar response and remission rates of $54 \%$ and $27 \%$, respectively. ${ }^{24} \mathrm{~A}$ meta-analysis of 4 RCTs in patients with ulcerative colitis demonstrated a $28 \%$ remission rate in patients treated with FMT compared with $8 \%$ in those who received placebo. ${ }^{23}$ FMT appears to have lower efficacy for IBD than for rCDI, suggesting that other factors are associated with IBD flares besides gut dysbiosis. ${ }^{25}$

Several reports have implied that IBD flares were induced by FMT that was administered for $\mathrm{CDI}^{2,26,27}$ A careful follow-up of patients with IBD treated with FMT is warranted because of this concern. An American Gastroenterology Association expert review, however, suggested that earlier FMT in patients with IBD and CDI might be recommendable owing to the relevant complication rate of CDI in these patients. ${ }^{28}$ Several RCTs in patients with Crohn's disease are currently under way. To date, there are no standardized practice guidelines for the use of FMT in IBD.

Besides rCDI and IBD, FMT is also being evaluated and considered an experimental treatment for other diseases including irritable bowel syndrome (IBS), ${ }^{29}$ nonalcoholic steatohepatitis, ${ }^{30}$ hepatic encephalopathy, ${ }^{31}$ obesity, ${ }^{32}$ and neurological diseases. ${ }^{33,34}$
Although donor screening is well accepted, recipient screening is controversial and without a consensus. Performing viral hepatitis, human immunodeficiency virus, and syphilis tests is recommended before FMT, so that if these diseases were to occur after FMT, clinicians would know that the disease was not transmitted from the donor to the recipient. ${ }^{21}$ Kelly et al., in a large case series drawn from multiple centers in the United States, showed that FMT seemed safe in immunocompromised patients, with the exception of neutropenic patients as a standard precaution. ${ }^{34}$

\section{DONOR SELECTION}

To minimize the risk of infection or other disease transmission, potential donors undergo rigorous screening including thorough history taking, serological tests, and fecal tests for parasitic, virologic, and bacterial pathogens. ${ }^{35}$ Although there are some variations between institutions, there are existing accepted protocols for donor screening (Tables 1,2 ). ${ }^{3,36,37}$

Donor stool is provided from 2 sources: patient-directed donors and universal donors through stool banks. ${ }^{38,39} \mathrm{~Pa}-$ tient-directed donors are identified by the recipients, usually

Table 1. Suggested Exclusion Criteria for Potential Stool Donors with a Risk of Infection or Microbiome-Associated Disease

\begin{tabular}{l}
\hline Exclusion criteria ${ }^{\text {a) }}$ \\
Age $<18$ yr or $>65 \mathrm{yr}$ \\
BMI $>30 \mathrm{~kg} / \mathrm{m}^{2}$ \\
Metabolic syndrome \\
Moderate to severe undernutrition \\
History of antibiotics use in the last 6 mo \\
Diarrhea within the last $3-6$ mo \\
History of Clostridium difficile colitis \\
Immune disorder or use of immunosuppressive medications \\
History of drug use or other recent risk factor for HIV or viral \\
hepatitis \\
History of travel to a tropical region in last 3 mo \\
Any gastrointestinal illness (IBD, IBS, gastrointestinal malignan- \\
cy, or major surgery) or complaints \\
History of autoimmune or atopic illness \\
History of chronic pain syndrome (fibromyalgia, chronic fatigue \\
syndrome) \\
Neurologic or neurodevelopmental disorders \\
History of malignancy \\
\hline $\begin{array}{l}\text { HIV, human immunodeficiency virus; IBD, inflammatory bowel } \\
\text { disease; IBS, irritable bowel syndrome. } \\
\text { a) Each institution can adopt different criteria. }\end{array}$
\end{tabular}


family members including spouses, siblings, children, or friends. Patient-directed donors are becoming less frequently used unless the recipient prefers a donor whose diet and other features are known, or if the recipient is concerned about transmissible agents that are perceived to exist in universal donors. The use of patient-directed donor stool incurs treatment delays owing to the time required for sourcing, screening, and testing donors, with resultant increased costs and scheduling problems. ${ }^{39,40}$ Additionally, using patient-directed donors may result in the donor feeling coerced and at a risk of revealing confidential information. ${ }^{41}$

Using universal donors for FMT has emerged as the most utilized method in the United States. Healthy volunteers who have a young age and a normal body mass index provide the stool after undergoing thorough history taking, physical examination, and serum and fecal pathogen screening tests. ${ }^{42}$

The use of universal donors has enabled a number of advances in FMT. Decreased microbial diversity is considered a possible cause of rCDI and other diseases of an altered microbiome. Using fecal material from multiple healthy donors could theoretically enhance the therapeutic efficacy of an in-

Table 2. Suggested Laboratory Tests for Potential Donors of Fecal Microbiota Transplantationa)

\begin{tabular}{lll}
\hline Tests & \multicolumn{1}{c}{ Blood } & \multicolumn{1}{c}{ Stool } \\
\hline Bacteria & Treponema & $\begin{array}{l}\text { Enteric pathogen culture: } \\
\text { Salmonella, Shigella, } \\
\text { Campylobacter } \\
\text { Helicobacter pylori EIA }\end{array}$ \\
& & VRE \\
Viruses & Hepatitis A virus IgM & Norovirus EIA or PCR \\
& Hepatitis surface antigen & Rotavirus EIA \\
& Anti-hepatitis C virus & \\
& HIV 1 and 2 & \\
Parasites & Entamoeba histolytica & Ovum and parasite \\
& Strongyloides stercoralis & $\begin{array}{l}\text { Microsporidia } \\
\text { Giardia fecal antigen/EIA }\end{array}$ \\
& & Cryptosporidium EIA \\
& & $\begin{array}{c}\text { AFB for Isospora } \text { and } \\
\text { Cyclospora }\end{array}$ \\
& & Clostridium difficile test \\
& & Toxin PCR
\end{tabular}

$\mathrm{AFB}$, acid-fast bacilli; CRP, C-reactive protein; EIA, enzyme immunoassay; ESR, erythrocyte sedimentation rate; HIV, human immunodeficiency virus; IgM, immunoglobulin M; PCR, polymerase chain reaction; VRE, vancomycin-resistant Enterococcus. a) The blood and stool tests should be completed within 1 month of donation, and the tests could be adopted differently depending on each institution and circumstance.

b) The test for Helicobacter pylori is usually needed in the case of upper gastrointestinal delivery. fusion or ingestion. An RCT on the application of FMT with multiple donors in ulcerative colitis showed clinical remission and endoscopic improvement as well as greater microbial diversity in the recepients. ${ }^{24}$ More studies are needed to confirm the value of multidonor FMT.

In addition, Lee et al. $^{43}$ conducted a noninferiority RCT of FMT with frozen and thawed stool in comparison with fresh stool for rCDI, to ensure the viability of microbes after freezing. They reported clinical resolution rates of $83.5 \%$ and $85.1 \%$ in each group, showing that frozen fecal material was as efficacious as fresh stool. Using frozen stool from universal donors reduces recipient costs as well as the time between the decision to perform FMT and the actual infusion. ${ }^{39,40}$

Owing to the cost-effectiveness and convenience of use of fecal material from universal donors, stool banks such as OpenBiome have emerged. OpenBiome uses strict protocols for the recruitment of healthy volunteers: the volunteers are screened, standardized products are generated, and the products are stored after freezing and can be delivered rapidly to 99\% of the entire United States. ${ }^{42}$ The additional advantages of stool banks are the ability to track registries and perform research on larger data obtained from multiple sites that conduct FMT, with the goal of assuring safety and efficacy. ${ }^{44}$ The U.S. Food and Drug Administration (FDA) has approved stool banks such as OpenBiome to provide fecal material for FMT for the treatment of $\mathrm{rCDI} .{ }^{45}$ For indications other than $\mathrm{rCDI}$, the FDA requires an investigational drug application. ${ }^{46}$

One of the main concerns about the use of stool banks is that multiple recipients could be adversely affected by a currently undetectable infection or transmissible process. OpenBiome has used extremely strict and detailed questionnaires to identify possibly risky donors in advance, and also to rescreen volunteers 60 days after the submission and before the release of the stool. Samples are also stored for future tracking. ${ }^{42}$

\section{PROCESSING OF FECAL MATERIAL}

Although there are trivial differences depending on the individual situation, most institutes prepare the stool based on the same protocol. The donor provides fresh stool within 1 month after screening. ${ }^{3}$ The potential donors collect their stool into a clean plastic bag and bring it to the microbiology laboratory. A minimum of $50 \mathrm{~g}$ of stool is needed. ${ }^{47}$ Then, the stool is diluted in normal saline, mixed in a sterile bag by hand stirring, and shaken or blenderized. It is then filtered through moistened 5-layer sterile gauze in a funnel and stored in a restricted safety cabinet to be delivered within $4 \mathrm{~h}$ of presentation to the endoscopy suite.

In the case of FMT with a universal donor, the fecal mate- 
rial is processed to $250-\mathrm{mL}$ aliquots in a similar standardized method and stored at $-80^{\circ} \mathrm{C}$ until delivered on dry ice to each requesting institute. $^{42}$

Fecal microbiota capsules could be prepared by concentration of the diluted, blended slurry, processed similarly. Then, the fecal solution is pipetted into a capsule holding $650 \mathrm{~mL}$ and then sealed into a second capsule. Commercially available acid-resistant, hypromellose capsules (DRcaps ${ }^{\mathrm{TM}}$; Capsugel, Cambridge, MA, USA) are usually utilized. A total of 30 capsules are prepared as a single therapeutic dose from each donor stool..$^{48}$ Capsules are also stored at $-80^{\circ} \mathrm{C}\left(-112^{\circ} \mathrm{F}\right)$ before use, maximally up to 6 months.

\section{PROCEDURE AND PATIENT MANAGEMENT}

It seems that FMT for rCDI is most efficacious in patients with mild to moderate disease that responded to antibiotics against $C$. difficile by the fourth day. ${ }^{11}$ In the other patients, the concern is that the diarrhea may be from another source. Some clinicians have recommended the earlier use of FMT for patients with severe or severe-complicated disease, as failure of standard therapy could result in higher morbidity and mortality. ${ }^{49}$ An accepted method of FMT is to provide antibiotics for at least 3 days before infusion to reduce the amount of C. difficile. ${ }^{20}$ Antibiotics are generally discontinued at 24-48 $\mathrm{h}$ before the FMT.

If the FMT is delivered by colonoscopy, bowel preparation is recommended to improve the visualization of the colon. In patients with a severe ileus, bowel preparation can be replaced by enemas or can be omitted. ${ }^{38}$ The standard dose of FMT is specific to each institution or physician; however, about 50 $100 \mathrm{~g}$ of donor fecal material that has been diluted to $250-500$ $\mathrm{mL}$ of infusate is most commonly used. .12,43,48 $^{-1}$

FMT can be administered either directly to the colon or from the upper gastrointestinal tract through capsule ingestion. ${ }^{38}$ Delivery to the colon is generally performed using colonoscopy, and less frequently through flexible sigmoidoscopy or an enema. Colonoscopic delivery has an efficacy of $84 \%-93 \%{ }^{41}$ and is the modality of choice according to published studies. ${ }^{47,50,51}$ If right-sided delivery of FMT is achieved using colonoscopy, the cure rate on a single infusion is $93 \%{ }^{9}$ The most serious risk that has been reported with respect to lower gastrointestinal tract administration is perforation. ${ }^{39,52}$ Theoretically, bleeding, adverse reaction to sedative drugs, cardiovascular events, transient fevers, or infections could occur, as with any colonoscopy procedure.

For patients with ileus, severe colitis, or objection to colonoscopy, FMT can be provided through the upper gastrointestinal tract via nasoenteric tubes, esophagogastroduodenoscopy, or capsule ingestion. ${ }^{38}$ The efficacy rates were reported to

Table 3. Analysis of Administration Modalities for Fecal Microbiota Transplantation

\begin{tabular}{|c|c|c|}
\hline Modality & Strength & Weakness \\
\hline Nasoenteric tube & $\begin{array}{l}\text { 1. No necessity for sedation } \\
\text { 2. Low cost }\end{array}$ & $\begin{array}{l}\text { 1. Discomfort associated with the administration } \\
\text { 2. Necessity for radiologic confirmation } \\
\text { 3. Risk of vomiting and aspiration }\end{array}$ \\
\hline Upper endoscopy & $\begin{array}{l}\text { 1. Safely performed in patients with a high risk for } \\
\text { colonoscopy complications }\end{array}$ & $\begin{array}{l}\text { 1. Same weaknesses as those of nasoenteric tube } \\
\text { 2. Procedure-related risk } \\
\text { 3. Necessity for sedation }\end{array}$ \\
\hline Capsule & $\begin{array}{l}\text { 1. Noninvasive } \\
\text { 2. More aesthetic appeal } \\
\text { 3. Cost- and time saving } \\
\text { 4. Convenience of administration }\end{array}$ & $\begin{array}{l}\text { 1. Large burden of the capsule } \\
\text { 2. Risk of vomiting and aspiration } \\
\text { 3. Cost }\end{array}$ \\
\hline Colonoscopy & $\begin{array}{l}\text { 1. Strong evidence of efficacy for rCDI } \\
\text { 2. Useful for differential diagnosis }\end{array}$ & $\begin{array}{l}\text { 1. Procedure-related risk } \\
\text { 2. Necessity for sedation } \\
\text { 3. Necessity for technical expertise } \\
\text { 4. Additional cost }\end{array}$ \\
\hline Sigmoidoscopy & 1. Can be preferred by patients & $\begin{array}{l}\text { 1. Procedure-related risk } \\
\text { 2. Inability to reach the right-sided colon }\end{array}$ \\
\hline Retention enema & $\begin{array}{l}\text { 1. Low cost } \\
\text { 2. Well tolerated } \\
\text { 3. No need for sedation and can be preferred by patients } \\
\text { 4. Can be easily repeated }\end{array}$ & $\begin{array}{l}\text { 1. Difficult to retain in some cases } \\
\text { 2. Inability to reach the right-sided colon } \\
\text { 3. Modality with the lowest efficacy }\end{array}$ \\
\hline
\end{tabular}

rCDI, recurrent Clostridium difficile infection. 
be between $81 \%$ and $86 \%{ }^{41}$ All forms of upper tract delivery increase the risk of vomiting or aspiration. ${ }^{53}$ Capsule delivery is the most recent modality of FMT. Capsules seem to be a reasonable choice for patients who have contraindications to colonoscopy, are geographically distant from an institution that performs colonoscopy, and are opposed to lower gastrointestinal tract access. Capsule delivery reduces the procedure time, colonoscopy cost, need for colon preparation, and risk of colonoscopy complications. ${ }^{54}$ Kao et al. demonstrated a comparable efficacy of capsule-delivered FMT to that of colonoscopy-delivered FMT. ${ }^{55}$ Although the standard dose per capsule is not yet defined, several studies have shown that a mean $1.6 \mathrm{~g}$ of stool per capsule yields a $70 \%$ cure rate without adverse events. ${ }^{48,56}$ Although no consensus has been achieved, the common impression by a number of authors is that colonoscopic administration has an about 5\%-10\% higher cure rate in $\mathrm{rCDI},{ }^{47,50,51}$ with the additional advantage of assuring that the fecal material reaches the colon because a water jet can be used to spray the material directly onto the mucosa. ${ }^{21}$ Table 3 summarizes the strengths and weakness of each modality.

The 2 most common side effects of FMT are bloating and loose stools for the first $24 \mathrm{~h}$, which usually resolve soon thereafter. ${ }^{35}$ Most patients generally have formed stool by 1-2 weeks. Stool testing for resolution is not recommended in those with formed stool, but is considered if 3 or more diarrhea stools per day occur after a few weeks. ${ }^{15,38}$ The polymerase chain reaction test for $C$. difficile toxin may remain positive for 30 days after a successful treatment, which is another reason not to test asymptomatic FMT recipients. ${ }^{38} \mathrm{~A}$ confusing situation is when abdominal cramping and intermittent frequent bowel movements occur in a patient who might be a carrier of $C$. difficile and who has received an FMT. Such patients most likely have post-infectious IBS. Therefore, the clinician should ideally be able to distinguish post-infectious IBS from rCDI to avoid unnecessarily repeating the FMT.

To date, there is no accepted standard protocol for follow-up. Most physicians and clinic staff contact the patient to assess treatment success and complications about 3-7 days after FMT. Another follow-up contact at 4-8 weeks is recommended. ${ }^{38}$

If the patient develops liquid stool and recurrence of symptoms with a positive stool test for C. difficile, the FMT is considered a failure. ${ }^{13} \mathrm{~A}$ recent study suggested that most failures occur within 4 weeks. ${ }^{49}$ Allegretti et al. ${ }^{57}$ reported that of the failed therapy cases, $25 \%$ failed within the first week and the patients are described as primary nonresponders. Another $61 \%$ failed between weeks 1 and 4 , with the patients referred to as early secondary nonresponders. The rest were consid- ered late secondary nonresponders. On the basis of these data, the authors suggested follow-up of patients approximately 4 weeks after the $\mathrm{FMT}^{57}$

Much of the concern about FMT arises from the fact that the long-term risks are unknown. Screening of donors by means of a thorough history taking may not reveal all future risks. Most FMT protocols endeavor to exclude donors with metabolic syndrome, obesity, neuropsychiatric disorders, and malignancies; however, a disease might emerge in the donor at a later date. This represents both a concern about FMT and a justification for the existence of stool banks, as follow-up of donors and maintenance of records would be more likely in stool banks, allowing the earlier identification of risks.

\section{CONCLUSIONS}

FMT is an established treatment for $\mathrm{rCDI}$, and is considered a second-line treatment. It is also being considered for other gastrointestinal diseases such as IBD, IBS, hepatic steatosis, and hepatic encephalopathy. Other disorders that may be related to gut dysbiosis, such as obesity, metabolic syndrome, autoimmune disorders, and neurological diseases, may also be improved by FMT. With stool banks providing universal door fecal material that has been highly screened and catalogued, barriers such as cost and availability can be overcome, allowing research and treatment to be simplified. Additionally, with the advent of capsule FMT, further increases in the use of this treatment may emerge with improved convenience, reduced patient reluctance, and simplified procedural preparation. To maintain patient safety and appropriate use of FMT, standardized protocols for donor screening, stool preparation, methods of delivery, and recipient indications for treatment are expected to emerge.

\section{Conflicts of Interest}

The authors have no financial conflicts of interest.

\section{REFERENCES}

1. Eiseman B, Silen W, Bascom GS, Kauvar AJ. Fecal enema as an adjunct in the treatment of pseudomembranous enterocolitis. Surgery 1958;44:854-859.

2. Chin SM, Sauk J, Mahabamunuge J, Kaplan JL, Hohmann EL, Khalili H. Fecal microbiota transplantation for recurrent Clostridium difficile infection in patients with inflammatory bowel disease: a single-center experience. Clin Gastroenterol Hepatol 2017;15:597-599.

3. Bakken JS, Borody T, Brandt LJ, et al. Treating Clostridium difficile infection with fecal microbiota transplantation. Clin Gastroenterol Hepatol 2011;9:1044-1049.

4. Brandt LJ, Aroniadis OC. An overview of fecal microbiota transplan- 
tation: techniques, indications, and outcomes. Gastrointest Endosc 2013;78:240-249.

5. Gerding DN. Metronidazole for Clostridium difficile-associated disease: is it okay for Mom? Clin Infect Dis 2005;40:1598-1600.

6. Wenisch C, Parschalk B, Hasenhundl M, Hirschl AM, Graninger W. Comparison of vancomycin, teicoplanin, metronidazole, and fusidic acid for the treatment of Clostridium difficile-associated diarrhea. Clin Infect Dis 1996;22:813-818.

7. Sadowsky MJ, Khoruts A. Faecal microbiota transplantation is promising but not a panacea. Nat Microbiol 2016;1:16015.

8. Choi HH, Cho YS. Fecal microbiota transplantation: current applications, effectiveness, and future perspectives. Clin Endosc 2016;49:257265.

9. Cammarota G, Ianiro G, Gasbarrini A. Fecal microbiota transplantation for the treatment of Clostridium difficile infection: a systematic review. J Clin Gastroenterol 2014;48:693-702.

10. Kelly CR, Khoruts A, Staley C, et al. Effect of fecal microbiota transplantation on recurrence in multiply recurrent Clostridium difficile infection: a randomized trial. Ann Intern Med 2016;165:609-616.

11. van Nood E, Vrieze A, Nieuwdorp M, et al. Duodenal infusion of donor feces for recurrent Clostridium difficile. N Engl J Med 2013;368:407-415.

12. Youngster I, Sauk J, Pindar C, et al. Fecal microbiota transplant for relapsing Clostridium difficile infection using a frozen inoculum from unrelated donors: a randomized, open-label, controlled pilot study. Clin Infect Dis 2014;58:1515-1522.

13. Surawicz CM, Brandt LJ, Binion DG, et al. Guidelines for diagnosis, treatment, and prevention of Clostridium difficile infections. Am J Gastroenterol 2013;108:478-498; quiz 499.

14. Trubiano JA, Cheng AC, Korman TM, et al. Australasian Society of Infectious Diseases updated guidelines for the management of Clostridium difficile infection in adults and children in Australia and New Zealand. Intern Med J 2016;46:479-493.

15. McDonald LC, Gerding DN, Johnson S, et al. Clinical practice guidelines for Clostridium difficile infection in adults and children: 2017 update by the Infectious Diseases Society of America (IDSA) and Society for Healthcare Epidemiology of America (SHEA). Clin Infect Dis 2018;66:987-994.

16. Quraishi MN, Widlak M, Bhala N, et al. Systematic review with meta-analysis: the efficacy of faecal microbiota transplantation for the treatment of recurrent and refractory Clostridium difficile infection. Aliment Pharmacol Ther 2017;46:479-493.

17. Moayyedi P, Yuan Y, Baharith H, Ford AC. Faecal microbiota transplantation for Clostridium difficile-associated diarrhoea: a systematic review of randomised controlled trials. Med J Aust 2017;207:166-172.

18. Fischer M, Sipe B, Cheng YW, et al. Fecal microbiota transplant in severe and severe-complicated Clostridium difficile: a promising treatment approach. Gut Microbes 2017;8:289-302.

19. Kelly CR, Kahn S, Kashyap P, et al. Update on fecal microbiota transplantation 2015: indications, methodologies, mechanisms, and outlook. Gastroenterology 2015;149:223-237.

20. Cammarota G, Ianiro G, Tilg H, et al. European consensus conference on faecal microbiota transplantation in clinical practice. Gut 2017;66:569-580.

21. Vindigni SM, Surawicz CM. Fecal microbiota transplantation. Gastroenterol Clin North Am 2017;46:171-185.

22. Zhang SL, Wang SN, Miao CY. Influence of microbiota on intestinal immune system in ulcerative colitis and its intervention. Front Immunol 2017;8:1674.

23. Costello SP, Soo W, Bryant RV, Jairath V, Hart AL, Andrews JM. Systematic review with meta-analysis: faecal microbiota transplantation for the induction of remission for active ulcerative colitis. Aliment Pharmacol Ther 2017;46:213-224.

24. Paramsothy S, Kamm MA, Kaakoush NO, et al. Multidonor intensive faecal microbiota transplantation for active ulcerative colitis: a randomised placebo-controlled trial. Lancet 2017;389:1218-1228.
25. Vindigni SM, Zisman TL, Suskind DL, Damman CJ. The intestinal microbiome, barrier function, and immune system in inflammatory bowel disease: a tripartite pathophysiological circuit with implications for new therapeutic directions. Therap Adv Gastroenterol 2016;9:606-625.

26. Khoruts A, Rank KM, Newman KM, et al. Inflammatory bowel disease affects the outcome of fecal microbiota transplantation for recurrent Clostridium difficile infection. Clin Gastroenterol Hepatol 2016;14:14331438.

27. Newman KM, Rank KM, Vaughn BP, Khoruts A. Treatment of recurrent Clostridium difficile infection using fecal microbiota transplantation in patients with inflammatory bowel disease. Gut Microbes 2017;8:303-309.

28. Khanna S, Shin A, Kelly CP. Management of Clostridium difficile infection in inflammatory bowel disease: expert review from the clinical practice updates committee of the AGA institute. Clin Gastroenterol Hepatol 2017;15:166-174.

29. Johnsen PH, Hilpüsch F, Cavanagh JP, et al. Faecal microbiota transplantation versus placebo for moderate-to-severe irritable bowel syndrome: a double-blind, randomised, placebo-controlled, parallel-group, single-centre trial. Lancet Gastroenterol Hepatol 2018;3:17-24.

30. Zhou D, Pan Q, Shen F, et al. Total fecal microbiota transplantation alleviates high-fat diet-induced steatohepatitis in mice via beneficial regulation of gut microbiota. Sci Rep 2017;7:1529.

31. Bajaj JS, Kassam Z, Fagan A, et al. Fecal microbiota transplant from a rational stool donor improves hepatic encephalopathy: a randomized clinical trial. Hepatology 2017;66:1727-1738.

32. Jayasinghe TN, Chiavaroli V, Holland DJ, Cutfield WS, O'Sullivan JM. The new era of treatment for obesity and metabolic disorders: evidence and expectations for gut microbiome transplantation. Front Cell Infect Microbiol 2016;6:15.

33. Ruggiero M. Fecal microbiota transplantation and the brain microbiota in neurological diseases. Clin Endosc 2016;49:579.

34. Kelly CR, Ihunnah C, Fischer M, et al. Fecal microbiota transplant for treatment of Clostridium difficile infection in immunocompromised patients. Am J Gastroenterol 2014;109:1065-1071.

35. Kelly BJ, Tebas P. Clinical practice and infrastructure review of fecal microbiota transplantation for Clostridium difficile infection. Chest 2018;153:266-277.

36. McCune VL, Struthers JK, Hawkey PM. Faecal transplantation for the treatment of Clostridium difficile infection: a review. Int J Antimicrob Agents 2014;43:201-206.

37. Brandt LJ. American Journal of Gastroenterology lecture: intestinal microbiota and the role of fecal microbiota transplant (FMT) in treatment of C. difficile infection. Am J Gastroenterol 2013;108:177-185.

38. Allegretti JR, Kassam Z, Osman M, Budree S, Fischer M, Kelly CR. The 5D framework: a clinical primer for fecal microbiota transplantation to treat Clostridium difficile infection. Gastrointest Endosc 2018;87:18-29.

39. Kim KO, Schwartz M, Gluck M. Reducing cost and scheduling complexity of fecal microbiota transplantation by using universal donor over patients-directed donors in patients with recurrent Clostrodium difficile infections. Gastroenterology 2018;154(6 Suppl 1):S191.

40. Edelstein C, Daw JR, Kassam Z. Seeking safe stool: Canada needs a universal donor model. CMAJ 2016;188:E431-E432.

41. Kassam Z, Lee CH, Yuan Y, Hunt RH. Fecal microbiota transplantation for Clostridium difficile infection: systematic review and meta-analysis. Am J Gastroenterol 2013;108:500-508.

42. OpenBiome. OpenBiome quality \& safety program [Internet]. Cambridge (MA): OpenBiome; c2017 [updated 2017 May; cited 2018 Apr 26]. Available from: https://www.openbiome.org/safety.

43. Lee CH, Steiner T, Petrof EO, et al. Frozen vs fresh fecal microbiota transplantation and clinical resolution of diarrhea in patients with recurrent Clostridium difficile infection: a randomized clinical trial. JAMA 2016;315:142-149.

44. Kazerouni A, Burgess J, Burns LJ, Wein LM. Optimal screening and donor management in a public stool bank. Microbiome 2015;3:75. 
45. Smith M, Kassam Z, Edelstein C, Burgess J, Alm E. OpenBiome remains open to serve the medical community. Nat Biotechnol 2014;32:867.

46. Gerding DN, Lessa FC. The epidemiology of Clostridium difficile infection inside and outside health care institutions. Infect Dis Clin North Am 2015;29:37-50

47. Gough E, Shaikh H, Manges AR. Systematic review of intestinal microbiota transplantation (fecal bacteriotherapy) for recurrent Clostridium difficile infection. Clin Infect Dis 2011;53:994-1002.

48. Youngster I, Russell GH, Pindar C, Ziv-Baran T, Sauk J, Hohmann EL. Oral, capsulized, frozen fecal microbiota transplantation for relapsing Clostridium difficile infection. JAMA 2014;312:1772-1778.

49. Fischer M, Kao D, Mehta SR, et al. Predictors of early failure after fecal microbiota transplantation for the therapy of Clostridium difficile infection: a multicenter study. Am J Gastroenterol 2016;111:1024-1031.

50. Postigo R, Kim JH. Colonoscopic versus nasogastric fecal transplantation for the treatment of Clostridium difficile infection: a review and pooled analysis. Infection 2012;40:643-648.

51. Sofi AA, Silverman AL, Khuder S, Garborg K, Westerink JM, Nawras A. Relationship of symptom duration and fecal bacteriotherapy in Clostridium difficile infection-pooled data analysis and a systematic review. Scand J Gastroenterol 2013;48:266-273.

52. Obi O, Hampton D, Anderson T, et al. Fecal microbiota transplant for treatment of resistant C. difficile infection using a standardized protocol: a community hospital experience: 2172 . Am J Gastroenterol 2014;109(Suppl 2):S629.

53. Baxter M, Ahmad T, Colville A, Sheridan R. Fatal aspiration pneumonia as a complication of fecal microbiota transplant. Clin Infect Dis 2015;61:136-137.

54. Zipursky JS, Sidorsky TI, Freedman CA, Sidorsky MN, Kirkland KB. Patient attitudes toward the use of fecal microbiota transplantation in the treatment of recurrent Clostridium difficile infection. Clin Infect Dis 2012;55:1652-1658.

55. Kao D, Roach B, Silva M, et al. Effect of oral capsule- vs colonoscopy-delivered fecal microbiota transplantation on recurrent Clostridium difficile infection: a randomized clinical trial. JAMA 2017;318:1985-1993.

56. Allegretti JR, Fischer M, Papa E, et al. Fecal microbiota transplantation delivered via oral capsules achieves microbial engraftment similar to traditional delivery modalities: safety, efficacy and engraftment results from a multi-center cluster randomized dose-finding study. Gastroenterology 2016;150(4 Suppl 1):S540.

57. Allegretti JR, Allegretti AS, Phelps E, Xu H, Fischer M, Kassam Z. Classifying fecal microbiota transplantation failure: an observational study examining timing and characteristics of fecal microbiota transplantation failures. Clin Gastroenterol Hepatol 2018;16:1832-1833. 